\title{
FDA-Zulassung zur wissenschaftlichen Erforschung des „Die Lunge klärenden und kühlenden Dekoktes" (Qingfei paidu tang) im Rahmen der COVID-19-Pandemie
}

Online publiziert: 26. Januar 2022

(c) The Author(s) under exclusive licence to Springer Medizin Verlag GmbH, ein Teil von Springer Nature 2022

Seit Beginn der Pandemie baut man in China in der Prophylaxe und Therapie von COVID-19 auf bewährte chinesische Rezepturen wie das „Die Lunge klärende und kühlende Dekokt"“ (Qingfei paidu tang, QFPDT). QFPDT stellt durch seine den o. pulmonalis (Fk „Lunge“, fei) befreiende und kühlende Wirkung einerseits sowie durch seine antiviralen Eigenschaften andererseits eine ideale Kombination bei diesem Krankheitsbild dar. Gerade gegenüber SARS-CoV-2 konnte auf molekularbiologischer Ebene eine hohe Spezifität dieser Rezeptur gezeigt werden, wodurch sich der therapeutische Effekt gut erklären lässt [1]. Aufgrund seiner klinischen Wirkung wurde es in China bereits im Jahr 2020 in die nationalen Leitlinien zur Behandlung von COVID-19 (,Diagnosis and Treatment guidelines of COVID-19“) aufgenommen und gilt weiterhin als eines der wichtigsten Mittel zur Behandlung dieser Erkrankung [2].

Die therapeutischen Erfolge konnten teilweise in Form von Studien belegt werden. Eine in China durchgeführte und international veröffentlichte retrospektive Beobachtung zeigt bei COVID-19-Patient*innen eine Verkürzung der symptomatischen Krankheitsdauer, gerade, wenn das Mittel wenige Tage nach Krankheitsbeginn eingenommen wurde [3]. In einer weiteren Studie zeigen sich erste Hinweise auf eine Reduktion der Mortalität, wobei einschränkend auf die retrospektive Methodik verwiesen sei [4].

Ausgehend von diesen positiven Resultaten hat nun im vergangenen Jahr die Forschergruppe um Dr. Andrew Shubov des Center for East-West-Medicine der University of California (UCLA) eine doppelblinde, randomisiert-kontrollierte Studie bei der Food and Drug Administration (FDA) angemeldet. In dieser Phase-I-Studie wird nun erstmalig prospektiv der Effekt einer leicht modifizierten Variante des „Die Lunge klärenden und kühlenden Dekoktes“ (mQFPDT) gegenüber einem Placebopräparat bei Patient*innen mit COVID-19 verglichen. Wie beim Studienleiter persönlich erfragt, handelt es sich beim mQFPDT im Wesentlichen um die Originalrezeptur, wobei Farfare flos $(\mathrm{Ku}-$ andonghua) aufgrund von Sicherheitsbedenken ersetzt wurde. Besonders hervorzuheben ist, dass sowohl Ephedrae herba (Mahuang) als auch Asari radix (Xixin) weiterhin Bestandteile der Studienrezeptur sind. Beide Substanzen sind vor allem in den USA seit geraumer Zeit aufgrund potentiell toxischer Implikationen so gut wie obsolet. Im Rahmen dieser Studie hat man jedoch dank konsequenter Qualitätskontrollen und enger Abstimmung des Herstellers mit der FDA von ebendieser die Genehmigung zur klinischen Durchführung erhalten. Die genaue Rezeptur inklusive täglicher Dosis wird erst mit den Ergebnissen veröffentlicht werden, frühestens also Ende 2022.

Für die Untersuchung werden seit Juli 2021 bis voraussichtlich Juli 2022 insgesamt 66 Teilnehmende eingeschlossen. Für den Einschluss in die Studie darf die Dauer von 72 Stunden seit Diagnosestellung bzw. 9 Tage seit Symptombeginn nicht überschritten werden. Nach Beginn der Studie werden die Teilnehmenden aufgefordert, über 14 Tage täglich dreimal 8 Kapseln des mQFPDT bzw. des Placebos einzunehmen. Das Hauptaugenmerk dieser Untersuchung liegt auf dem Sicherheitsprofil und der Verträglichkeit des Präparats sowie der generellen Machbarkeit einer möglicherweise künftigen, größer angelegten Studie. Als sogenannte sekundäre Endpunkte werden auch klinische Daten bezüglich des Krankheitsverlaufs und verschiedene Biomarker wie etwa Entzündungswerte erhoben [5].

Vor dem Hintergrund der sehr aufwendigen Zulassungsverfahren von Phytotherapie-Studien und der häufig resultierenden Machbarkeitsbeschränkungen geht von der FDA-Genehmigung zur Studiendurchführung ein hoher Symbolwert aus. Die Genehmigung von Ephedrae herba (Mahuang) und sogar Asari radix (Xixin) als Bestandteile der Studienrezeptur zeigt hier im Positiven, dass Verbote 
und Einschränkungen unter gewissen Voraussetzungen auch vorübergehend sein können. Obwohl es abzuwarten bleibt, welche Ergebnisse in der Untersuchung erzielt werden können, besteht bereits heute eine sehr positive empirische Erfahrung mit dieser Rezeptur. Besonders hilfreich erscheint hierbei die vorgefertigte Darreichungsform in Form eines Sirups [6], zeigt doch die rasche Verfügbarkeit als wichtige Voraussetzung einer zeitnahen Einnahme einen wesentlichen klinischen Vorteil.

Moritz Hempen

\section{Literatur:}

1. Li LDZ, et al. Potential Targets for Treatment of Coronavirus Disease 2019 (COVID-19): A Review of Qing-Fei-Pai-Du-Tang and Its Major Herbs. Am J Chin Med. 2020;48(5):1051-71.

2. Li S, et al. Traditional Chinese Medicine as Potential Therapy for COVID-19. Am J Chin Med. 2020;48(6):1263-77.

3. Nannan Shi et al. Association between early treatment with Qingfei Paidu decoction and favorable clinical outcomes in patients with COVID-19: A retrospective multicenter cohort study. Pharmacol Res. 2020;161:105290.

4. Zhang L, et al. Association between use of Qingfei Paidu Tang and mortality in hospitalized patients with COVID-19: A national retrospective registry study. Phytomedicine. 2021;85:153531.

5. FDA Approved Modified Qing Fei Pai Du Tang for UCLA/UCSD double blinded randomized controlled trial.. https://clinicaltrials. gov/ct2/show/NCT04939415.

6. Die Lunge kühlendes und klärendes Dekokt.. www.qinax.com. 